\title{
A Survey: Ad-hoc on Demand Distance Vector (AODV) Protocol
}

\author{
Meeta Singh, $\mathrm{PhD}$ \\ Manav Rachna International University, Faridabad
}

\author{
Sudeep Kumar \\ Govt. Polytechnic for Women, Faridabad
}

\begin{abstract}
The main objective of this paper is to provide background information for understanding of Ad-hoc on demand distance vector (AODV) protocol. This paper provides a critical review of literature on mobile ad-hoc networks and the routing protocols. This paper will discusses the concept and characteristics of mobile ad-hoc networks. The features and classification of the ad-hoc routing protocols \& explains the description of Ad-hoc on demand distance vector (AODV) protocol with its mechanisms. This paper also discusses the link failure in mobile ad-hoc networks and the link state prediction is illustrated.
\end{abstract}

\section{Keywords}

Mobile ad-hoc network, AODV, protocol, Link, failure

\section{INTRODUCTION}

Mobile Ad-Hoc Networks A mobile ad-hoc network is considered as a special type of wireless networks which contains a collection of wireless devices such as tablets, iPad, and laptops. These devices organize a temporary network without depending on fixed infrastructures. Basically, mobile ad-hoc networks are peer to peer multihop mobile wireless networks according to which each packet information is sent from the source to the destination node through an intermediate node as demonstrated in Figure 1.

The communication of mobile nodes with each other is performed through the wireless medium. Mobile ad-hoc networks may operate independent or with few selected routers to communicate with an infrastructure network such as the Internet as mentioned in [1]. The topology of mobile adhoc network is dynamic because the mobile nodes can leave or join the network at anytime and anywhere. According to Das et al. [2] one of the major advantages of mobile ad-hoc networks is the attractive choice for military field. However, the freedom and flexibility of mobile ad-hoc networks emerge with some complication and challenges. The lack of preexisting infrastructure, frequently changing topology, multihop nature, bandwidth constraints, energy consumption, and network scalability, add the challenges and complexities to the traditional of wireless networks.

\section{Mobility and Network Topology}

Node mobility is the main factor that affect the performance of MANET as stated in Tonguz [3] and Lenders [4]. The fast unpredictable movement of the intermediate nodes in MANET environment dynamically change the network topology. This in turn causes a break in the discovered communication links. When the links are disrupted, the result is dropping of large amount of the data packets that were sent to the next hop during the forwarding process. As a result, the rnetwork forces the underlying protocol to recover the failed links or starts discovering new routes to continue transmission the data packets. However, the increase of many activities in mobile ad-hoc networks, causes a frequent exchange of routing information over the bandwidth constrained communication channel. According to Bandana [5], this leads to increase in overall routing overhead of the network.
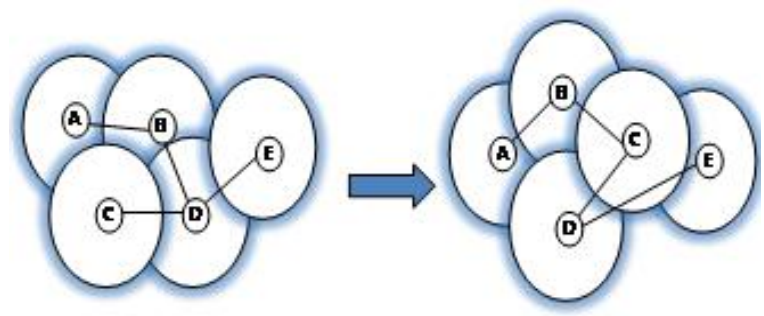

:Transmission range

:Mobile node :Link

Figure 1: Dynamic Topology in Mobile Ad-Hoc Networks

Figure 2 illustrates the dynamic changes of topology under node movement and multi-hop nature of mobile ad-hoc networks. Figures 2 (a) and 2 (b) represent the network topology at time $t$ and $t+1$ respectively where the mobile node (6) presents the source and mobile node (1) presents the destination.

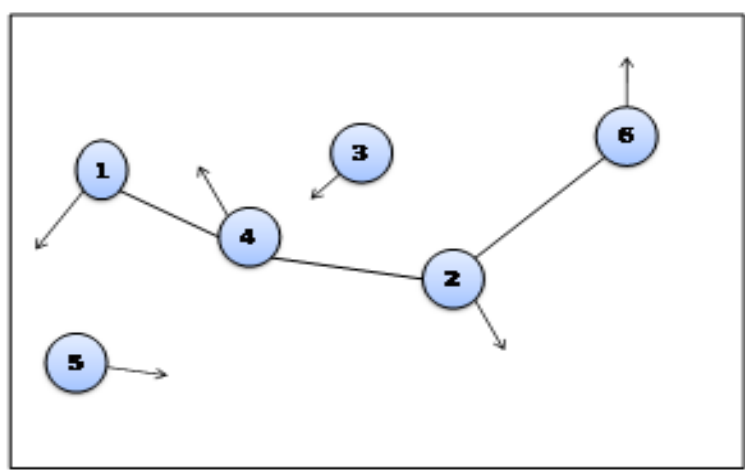

(a) Network state at time $t$

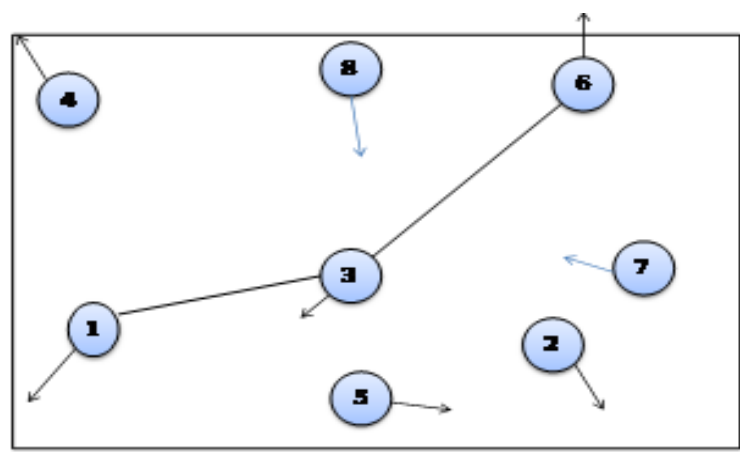

(b) Network state at time $\mathbf{t}+1$

Figure 2: Dynamically Changing Network Topology a) Network state at time $t$ b) Network state at time $t+1$ 
(a) Effect of Mobility and Link Stability

Mobility directly affects number of the link facts such as the number of established links, number of disrupted links and the duration of links in MANET as mentioned in Lenders et al. [4]. As a result, the amount of data packets that can be carried over the wireless link is reduced. Mobility of the individual mobile node causes a dynamic change of the network topology, thereby prompting the routing protocol to perform network reconfiguration continuously. Furthermore, mobility affects the end-to-end performance measurements such as network throughput, the amount of control routing overhead, packet delivery ratio, average delay, and allocation of resources as stated in Mullen and Huang [6]. High node mobility makes the communication route unstable due to occurrence of more link breakages within the communication route. In addition, the wireless links might also be broken due to various sources of signal interference and the packet collision. It has been discovered that the node mobility and interference/collision have totally different effects on the lifetime of the routes as stated in Lenders et al. [4].

\section{ROUTING PROTOCOLS IN MOBILE AD-HOC NETWORKS}

The primary goal of using routing protocols in ad-hoc networks is to discover and establish routes between mobile nodes to send the data packets from the source to destination so that messages will be delivered timely as stated in Saito et al. [7]. Routing protocols for mobile ad-hoc networks usually call for mobility management and scalable design. Mobility management is performed by exchanging the routing information between moving hosts in mobile ad-hoc networks. However, frequent information exchanges can be costly in terms of bandwidth and power consumption. By minimizing the frequent information changes, these costs will be decreased. The scalable design works for the large size networks which needs both routing protocols and resource consumption to be scalable. Designing a reliable and efficient routing protocol is very challenging in MANET due to the dynamic topology that results from mobility of nodes and limited network bandwidth as well as different types of wireless communication restraints as mentioned in [8, 9]. These restraints are variable link quality, energy constrained nodes, interference, and exposed terminal problems. However, the issues of the routing protocol performance in mobile ad-hoc networks are divided into two areas. The first area focuses on the limitation of the environments such as wireless, limited bandwidth, power consumption, and security. The second area concerns about the way of transferring the data communication which might take place leading to the desirable qualitative features of mobile ad-hoc routing protocols as stated in [10]. The MANET working group determines some desirable qualitative properties of adhoc routing protocol as stated in RFC 2501 [11].

\section{CLASSIFICATION OF MOBILE AD- HOC ROUTING PROTOCOLS}

The classification of ad-hoc routing protocols can be divided according to their approaches for creating and maintaining routes. These protocols can be classified into proactive, reactive, and hybrid. The classification depends on the reaction of the mobile nodes in the routing determination process as stated in Djenouri et al. [12]. Figure 3 shows the taxonomy of ad-hoc routing protocols.

Proactive Routing Approach

In this category of the routing protocols, the route is always available for the data communication from the source node to the destination node. Every mobile node in proactive routing approach keeps updating the routing information periodically in its routing table. The main advantage of table driven routing approach is when the source node requires to send the data packets to a desired destination. The route is already available and there is no latency for establishing the routes. On the other hand, the disadvantage of this approach is some routes in some situations may never be used for communication which leads to consume network bandwidth and node energy.

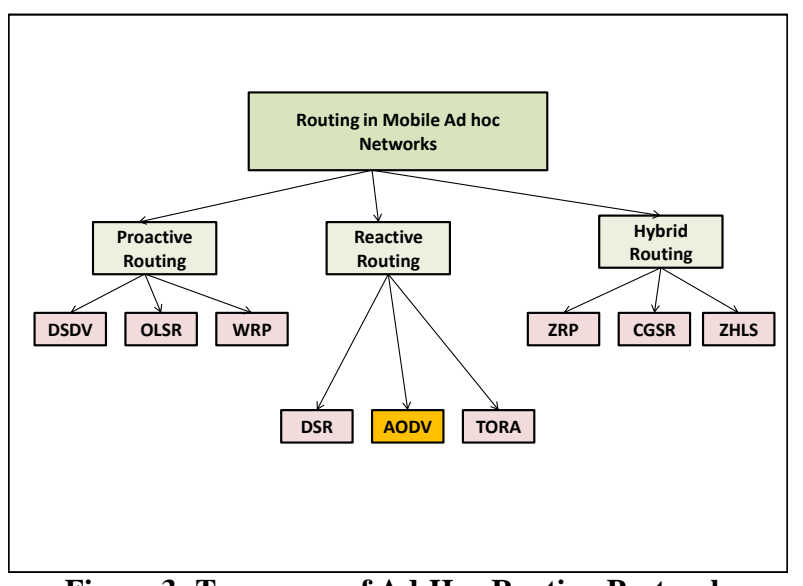

Figure 3: Taxonomy of Ad-Hoc Routing Protocols

The most popular proactive routing protocols are the Destination Sequenced Distance Vector Routing (DSDV), Optimized Link State Routing Protocol (OLSR) and Wireless Routing Protocol (WRP).

Reactive Routing Approach

Reactive routing protocols are also called "On-Demand Routing". On-demand routing protocols means that a route is established exclusively when the data packets are in need to be delivered to the destination. On-Demand routing protocols takes a different approach for routing than table driven protocols such as Temporally Ordered Routing Algorithm (TORA) , Ad hoc On-Demand Distance Vector Routing (AODV), and Dynamic Source Routing (DSR). The routes to the destination are discovered when they are actually needed. When a source node requires to send data packets to the destination, it starts checking the routing table to determine whether it has a route to the destination or not. If there is no existing route, the source node starts route discovery procedure in order to find a path to the destination.

Reactive routing protocols are based on Distance Vector concept (DV) which can significantly decrease the routing overhead and the power consumption. These protocols do not need to keep searching and maintaining the routes because there is no data traffic to send as stated in Royer and Toh [8].

The works in $[3,8,9,10,13,14]$ have evaluated the comparison of proactive and reactive with multi-hop routing protocols such as DSDV, TORA, DSR, ABR, and AODV. Through their simulation results, they have observed that the reactive routing protocols outperform proactive protocols in terms of packets delivery ratio, routing overhead, energy efficiency, and stability. 


\section{LINK FAILURE IN MOBILE AD- HOC NETWORKS}

The routes between the source and destination pair in mobile ad-hoc networks frequently become unavailable due to link failure that occurs somewhere on the route. The link failure arises from many reasons, such as node mobility, power consumption, fading in the communication channel and errors in the noisy wireless medium. Thus, mobile ad-hoc networks are normally highly dynamic and the routing algorithms should be developed in order to deal with link failures effectively.

According to [15], there are two kinds of failures in MANET: node failure, and link failure. The link failure occurs more often than node failure because the node failure usually carries multiple link failures since the link will fail on a failed node. The link failure is classified into two types in terms of the number of broken links: single link failure and multiple link failure. These failures can be classified based on the recovery time from the link failure, into permanent, transient or intermittent failure as described in $\mathrm{Wu}$ et al. [16]. Permanent failure occurs when the failure can not be repaired automatically within a period of time. Permanent failures, that indefinitely make the path non operational are frequently found due to the physical damages of the node, battery depletion or long term malicious attack. In transient failure or intermittent, this type of failure occurs for short periods of time. A transient failure is more frequent than permanent failure.

This research focuses on transient (or intermittent) link failures that are caused by node mobility and which are quite common in mobile ad-hoc networks. How to deal with the failure of routing efficiently and reliably is an extremely necessary part of wireless ad-hoc routing protocol research. The link or node failures, which make the paths unreliable either because they effected the link or because the node cannot perform its job for forwarding the incoming packet, are normally experienced in mobile ad-hoc routing protocol research. These failures results in packet loss and increases the end-to-end delay in the network. Figure 4 shows an example of link failure in mobile ad-hoc networks.

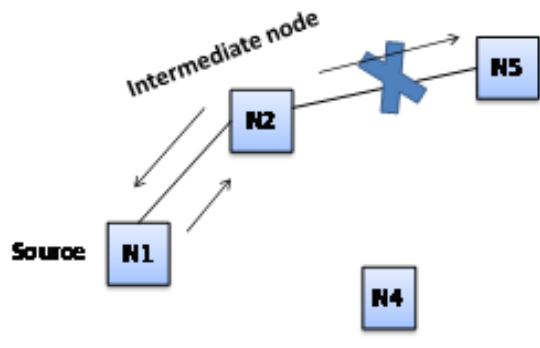

Figure 4: Example of Link Failure in MANET

\section{LINK STATE PREDICTION}

The link state prediction is an approach used to predict the link failure in advance. There are three main prediction methods used to predict the link status for the near future as stated in Aguayo et al. [17]. These are:

- Signal strength-based methods: This method attempts to predict the link breakage based on the received signal strength from the predecessor node as mentioned in $[18,19,20,21]$. Each signal power keeps a minimum threshold and compares the received signal strength with this value. If the received signal is less than the threshold value, then the link to the next hope will be disconnected soon. In this aspect, the current node tries to find an alternative route to forward the data flow to the desired destination. The signal strength-based method has a good performance in any environment, especially in urban environments.

- Beacon packet-based methods: In this method, each mobile node broadcasts a Hello message to its next hop neighbors where it introduces itself to them and knows them. In some cases of the beacon packet based method, two mobile nodes receive a minimum threshold number of Hello messages from each other. In this respect, it can be concluded that they have stable state relatively. Therefore, in the route discovery process, they try to select such mobile nodes to determine the stable routes. Finding stable routes can postpone the link breakage as far as possible. This method suffers from the stale routes in the route cache as written by Heissenbuttel et al. [22]. However, to update the routing information in the route cache, this method has to reduce the broadcasting period as mobile nodes speed increases. This results in a large routing overhead in the network and this overhead may become larger than the overhead of underlying routing protocol invocation to find a new route in some scenarios.

- Position information-based methods: This method depends also on the packet received signal in order to compute its distance from the previous hop and accordingly takes an action. If the distance becomes larger than the maximum allowable threshold, then the current node considers the link breaks soon. In this respect, the new route discovery process is initiated in order to find a new route before the current one fails. This method requires a positioning system like Global Positioning System (GPS) to estimate two consecutive nodes distance. This method has good performance in open areas, whereas in urban and similar environments are not too precise. As result, the mobile nodes may fail in their prediction process as stated in [21]. However, based on the three methods above, it seems that the 
signal strength based method is more suitable than the two other methods in terms of suitability to urban areas as well as the power consumption. Hence, this research utilizes the first method (signal strength based method).

\section{AD HOC ON-DEMAND DISTANCE VECTOR ROUTING (AODV)}

In Ad hoc On-demand Distance Vector Routing (AODV) to find a route to the destination, the source broadcasts a route request packet. This broadcast message propagates through the network until it reaches an intermediate node that has recent route information about the destination or until it reaches the destination. When intermediate nodes forwards the route request packet it records in its own tables which node the route request came from. This information is used to form the reply path for the route reply packet as AODV uses only symmetric links. As the route reply packet traverses back to the source, the nodes along the reverse path enter the routing information into their tables. Whenever a link failure occurs, the source is notified and a route discovery can be requested again if needed. It is based on standard Distance Vector Algorithm. Nodes maintain route cache and uses destination sequence number for each route entry does nothing when connection between end points is still valid Route Discovery Mechanism is initiated when a route to new destination is needed by broadcasting a Route Request Packet (RREQ).Route Error Packets (RERR) is used to erase broken Links.
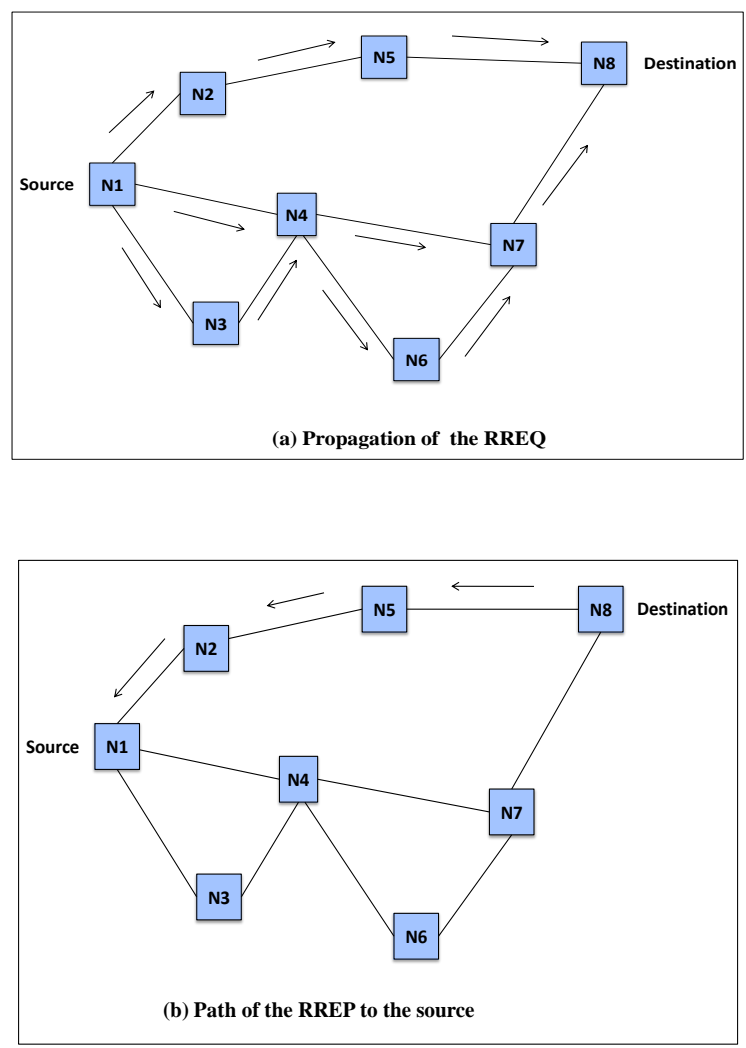
Figure 5: AODV Protocol (a) Propagation of RREQ, (b)
Path of the RREP to the source

When the network entity needs to send a message to another node, it calls upon AODV to determine the next-hop. Whenever an AODV router receives a request to send a message, it checks its routing table to see if a route exists.
Each routing table entry consists of the following fields:

- Destination address

- Next hop address

- Destination sequence number

- Hop count

If a route exists, the router simply forwards the message to the next hop. Otherwise, it saves the message in a message queue, and then it initiates a route request to determine a route. The following flow chart illustrates this process:

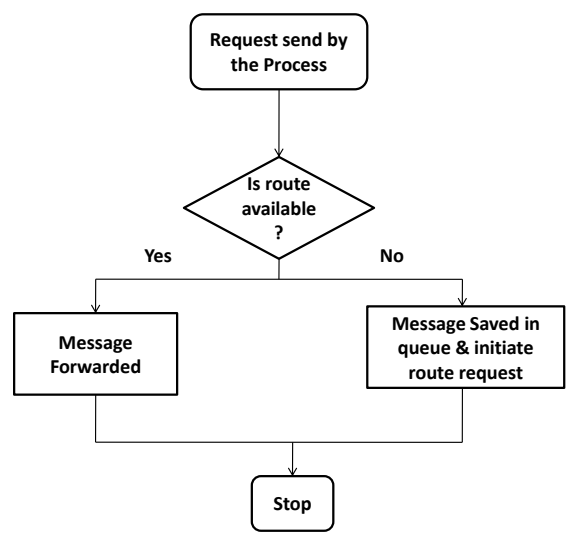

Figure 6: AODV

Upon receipt of the routing information, it updates its routing table and sends the queued message(s).AODV nodes use four types of messages to communicate among each other. Route Request (RREQ) and Route Reply (RREP) messages are used for route discovery. Route Error (RERR) messages and HELLO messages are used for route maintenance.

\section{(a) Route Discovery Mechanism of AODV Protocol}

In the flooding protocol, when a node originates or forwards a route request message to its neighbors, the node will likely receive the same route request message back from its neighbors. To prevent nodes from resending the same RREQs (causing infinite cycles), each node maintains a route request buffer, which contains a list of recently broadcasted route requests. Before forwarding a RREQ message, a node always checks the buffer to make sure it has not already forwarded the request. RREQ messages are also stored in the buffer by a node that originates a RREP message.

Each destination (node) maintains a monotonically increasing sequence number, which serves as a logical time at that node. Also, every route entry includes a destination sequence number, which indicates the "time" at the destination node when the route was created. The protocol uses sequence numbers to ensure that nodes only update routes with "newer" ones. Doing so, we also ensure loop- freedom for all routes to a destination. All RREQ messages include the originator's sequence number, and its (latest known) destination sequence number. Figure 7 summarizes the action of an AODV node when processing an incoming message.

In an ad hoc network, links are likely to break due to the mobility of the nodes and the ephemeral nature of the wireless channel. Hence, there must be a mechanism in place to repair routes when links within active routes break. An active route is defined to be a route that has recently been utilized for the transmission of data packets. When such a link break occurs, 
the node upstream of the break (i.e., the node closer to the source node), invalidates in its routing table all destinations that become unreachable due to the loss of the link. It then creates a Route Error (RERR) message, in which it lists each of these lost destinations.

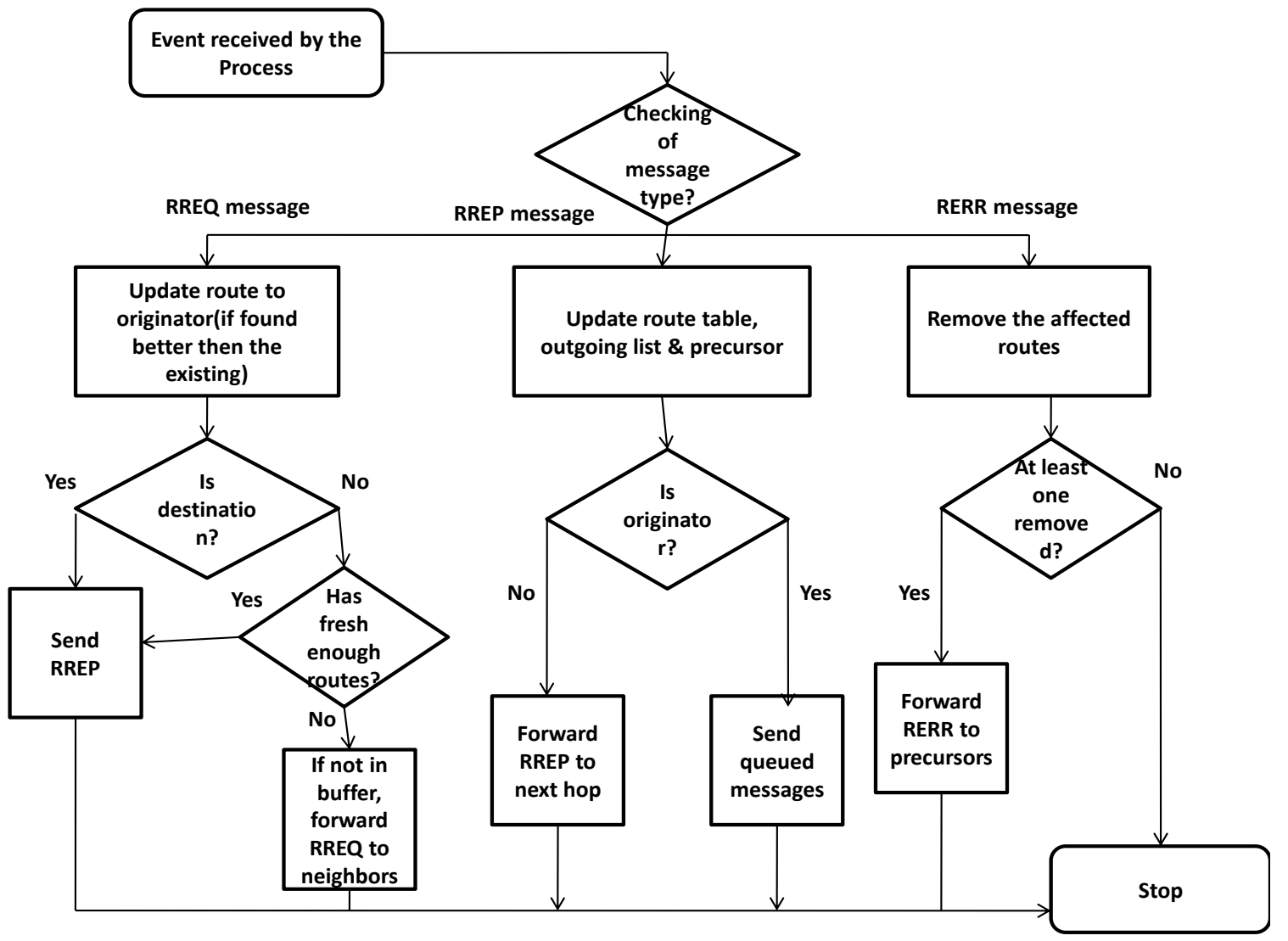

Figure 7: Flow chart Route Request

(b) Route Maintenance Mechanism of AODV Protocol

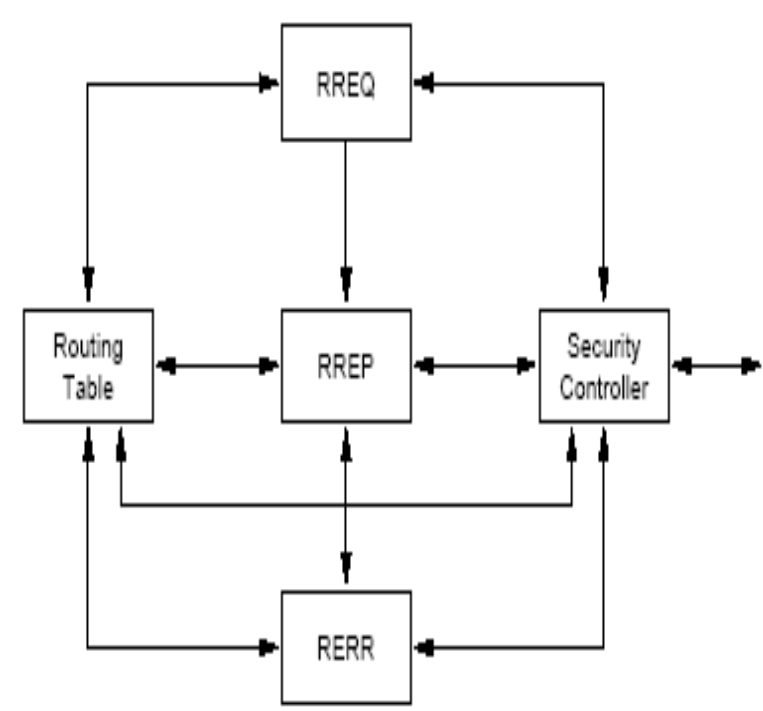

Figure 8: Route Reply in AODV

The node sends the RERR upstream towards the source node. If there are multiple previous hops that were utilizing this link, the node broadcasts the RERR; otherwise, it is unicast. In the Figure 9, the link between nodes N2 and N5 on the path from Source to Destination is broken. Node N2 invalidates its route table entries for both nodes N5 and N8, creates a RERR message listing these nodes, and sends the RERR upstream towards the source. When a node receives a RERR, it first checks whether the node that sent the RERR is its next hop to any of the destinations listed in the RERR. If the sending node is the next hop to any of these destinations, the node invalidates these routes in its route table and then propagates the RERR back towards the source. The RERR continues to be forwarded in this manner until it is received by the source. Once the source receives the RERR, it can re-initiate route discovery if it still requires the route.

\section{(c) Route Cache of AODV Protocol}

This has been observed that AODV finds new routes by making a route request broadcast which travels through various intermediate nodes before reaching the destination node. These requests carry a lot of information about the network topology as they pass through different node but due to lack of caches at intermediate nodes, this information cannot be tapped by the nodes to be use later. So by providing all the nodes with an extra cache and by making changes in the RREQ packets such as to enable them to carry the information about the nodes through which the byepass, intermediate nodes can save the information about the network topology contained in the RREQ packets. This reduces the time and overhead to find new routes in cases of route failure. From now no, we will call the AODV with cache enabled as AODV-WC and AODV without caching as AODV-WOC. 


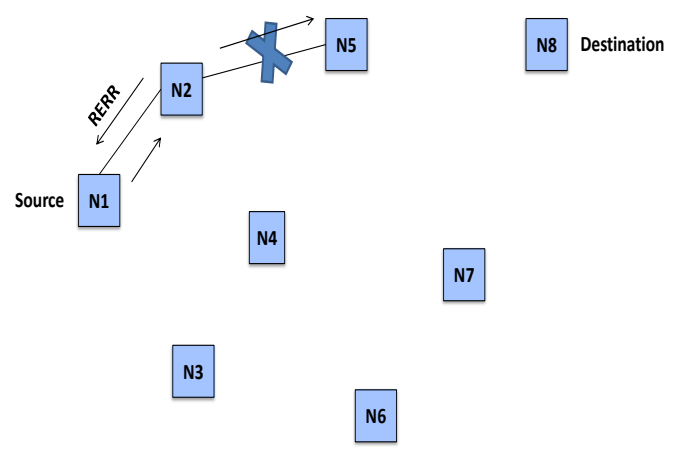

Figure 9: Link breaks Notification

Each node now has a separate queue (apart from the queue which AODV has for maintaining routing information) which acts as a cache for the routes. For this purpose, we have used the same queue structure which AODV uses for maintaining its routes. To reduce the problem of stale caching, a cache timer is introduced in the caches and an appropriate cache timeout value is found to get the maximum efficiency from the cache even in the case of high mobility (low pause time). So any route that does not get updated within the cache timeout period from the time of its addition to the cache, is discarded as stale Route request packets (RREQ) should be able to carry the node addresses and latest sequence numbers (It is the same sequence number as used by AODV to check the freshness of a route) of the intermediate nodes they have passed before reaching the destination node. For this purpose, we have implemented a special data structure in the AODV RREQ packet header which forms a link list of node addresses and sequence numbers of the nodes through which the packet has crossed All the nodes on receiving a route request packet should, apart from doing their already specified tasks, read the node addresses and sequence numbers in the packet and add them to their caches as the nodes reachable from the last node through which the packet is coming. Then before broadcasting the packet to the neighboring nodes, the nodes should append their own address and a latest unused sequence number into the packet.

\section{CONCLUSION}

In the last years, lots of people are pushed to study the performance in wireless networks because of the increasingly use of wireless networks. Some researcher's or users advices to change routing protocols to dynamic protocols or source routing protocol. But there are some researcher's make a difference of the cause of packets lost or they can change the congestion control protocol of TCP. This paper provides the survey on mobile adhoc network protocol AODV.

\section{REFERENCES}

[1] Lee and D.H. Cho, "A new adaptive routing scheme based on the traffic characteristics in mobile ad hoc networks," In Proc. Of Fall VTC 2000, September 2000, 2911-2914.

[2] B. Das, V. Bharghavan, Routing in ad-hoc networks using minimum connected dominating sets, in: Proceeding of the IEEE International Conference on Communication (ICC97), June 1997
[3] M. Bhatt, R. Chokshi, S. Desai,S. Panichpapiboon, N. Wisitpongphan and O. K. Tonguz "Impact of Mobility on the Performance of Ad Hoc Wireless Networks", IEEE Vehicular. Technology Conference (VTC 2003), Orlando, FL, October 2003.

[4] V. Lenders, J. Wagner, and M. May, "Analyzing the Impact of Mobility in Ad Hoc Networks", REALMAN'06, May 26, 2006, Florence, Italy.

[5] Paudel, Bandana, and Ivan G. Guardiola. On the Effects of Small-Scale fading and Mobility in Mobile Wireless Communication Network. Rolla: Graduate School of Missouri S\&T, July 2009.

[6] J. Mullen, H. Hong. "Impact of Multipath Fading in Wireless Ad Hoc Networks." 2nd ACM Workshop on Performance Evaluation of Wireless Ad Hoc, Sensor, and Ubiquitous Networks (PE-WASUN ${ }^{\mathrm{e}} 05$ ). October 10-13, 2005.

[7] M. Aoki, M. Saito, H. Aida, and H.Tokuda. ANARCH: A Name Resolution Scheme for Mobile Ad Hoc Networks. In Proceedings of the 17th International Conference on Advanced Information Networking and Applications (AINA '03), pages 723 730, Xi'an, China, March 2003.

[8] D.O. Jörg, Performance Comparison of MANET Routing Protocols in Different Network Sizes, Comp. Science Project, Institute of Comp. Science and Networks and Distributed Sys, University of Berne, Switzer land (2003)

[9] S. Murthy and J.J. Garcia-Luna-Aceves, "An efficient routing protocol for wireless networks," ACM Mobile Networks and Applications Journal, 1996.

[10] Josh Broch et al., "A performance comparison of multihop wireless ad hoc network routing protocols", In Proc. the 4th annual ACM/IEEE international conference on Mobile computing and networking, MobiCom '98, October 25-30, 1998, Dallas, Texas, USA

[11] S. Corson, J. Macker, " Mobile Ad hoc Networking (MANET): Routing Protocol Performance Issues and Evaluation Considerations", January 1999

[12] DJENOURI and Nadjib BADACHE. "An energy efficient routing protocol for mobile ad hoc network. In The second proceeding of the Mediterranean Workshop on Ad-Hoc Networks, Med-Hoc-Nets 2003, Mahdia, Tunisia, pages 113\{122, 25-27 June 2003.

[13] Singh, Meeta, and Jigyasa Sharma. "Performance analysis of secure \& efficient AODV (SE-AODV) with AODV routing protocol using NS2." Reliability, Infocom Technologies and Optimization (ICRITO)(Trends and Future Directions), 2014 3rd International Conference on. IEEE, 2014.

[14] Singh, Meeta, and A. K. Yadav. "Performance Evaluation of AODV and DSR using Random Way Point Mobility Model." International Journal of Computer Applications 46.19 (2012).

[15] Josh Broch, David B. Johnson, and David A. Maltz. The Dynamic Source Routing Protocol for Mobile Ad Hoc Networks. Internet-Draft, draft-ietf-manet-dsr-03.txt, October 1999. Work in progress. Earlier revisions published June 1999, December 1998, and March 1998 
[16] I. Chlamatac, M. Conti, and J. Liu "Mobile Ad Hoc Networking: Imperatives and Challenges"; Ad Hoc Networks 1 (2003) 13-64.

[17] Y. Wang, Y. H. jin, W. Guo, W. Q. Sun, W. S. hu and M. Y. Wu, (2007) " Joint Scheduling for Optical Grid Applications", Journal of Optical Networking, Vol. 6, pp. 304-318.

[18] D. S. J. D. Couto, D. Aguayo, J. Bicket, and R. Morris, "A High Throughput Path Metric for Multi-hop Wireless Routing”, Proc. of MobiCom-2003, pp. 134-146, 2003.

[19] Y. Qi and H. Kobayashi, "A Unified Analysis for Cramer-Rao Lower Bound for Geolocation," Proc. 36th
Annual Conference on Information Sciences and Systems (CISS 2002), Princeton University, March 2124, 2002.[33]

[20] J. Caffery, Wireless Location in CDMA Cellular Radio System, Kluwer Academic Publisher, 1999

[21] ] H. L. Van Trees, Optimum array processing: Part IV of detection, estimation and modulation theory, John Wiley \& Sons, Inc., 2002

[22] M. Heissenbüttel and T. Braun, "BLR: A beacon-less routing algorithm for mobile ad-hoc networks," Institute of Computer Science and Applied Mathematics (IAM), Tech. Rep., March 2003. 